\title{
Why Does Wood Not Get Contact Charged? Lignin as an Antistatic Additive for Common Polymers
}

\author{
Mertcan Özel, ${ }^{\S}$ Fatma Demir, ${ }^{\S}$ Aizimaiti Aikebaier, Joanna Kwiczak-Yiguitbaşı, H. Tarik Baytekin, \\ and Bilge Baytekin*
}

Cite This: Chem. Mater. 2020, 32, 7438-7444

Read Online

ABSTRACT: Contact electrification (CE), or the development of surface charges upon contact and separation, is a millennia-old scientific mystery and the source of many problems in the industry. Since the 18th century, efforts to understand $\mathrm{CE}$ have involved ranking materials according to their charging propensities. In all these reports, wood, an insulator, turns out to be surprisingly immune to CE. Here, we show that this unique antistatic nature of wood is attributable to its lignin content, i.e., lignin removal from wood ceases the antistatic property, and (re)addition brings it back. The antistatic action of lignin (also an insulator) is proposed to be related to its radical scavenging action and can be explained through the bond-breaking mechanism of CE. Our results also show that lignin, a sustainable, low-cost
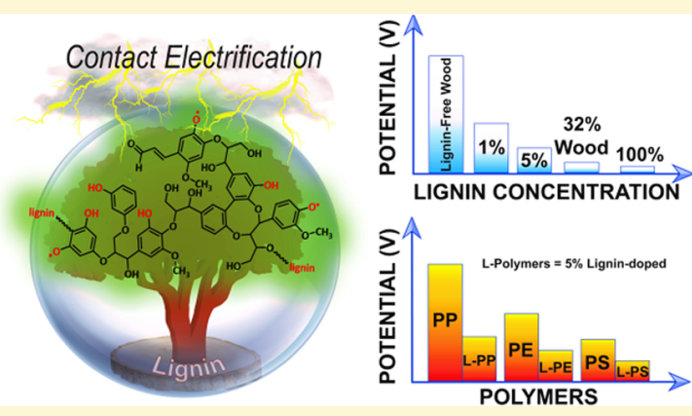
biopolymer, can be used as an antistatic additive in some representative examples of elastomers and thermoplastics, displaying the universal nature of its antistatic action.

$\mathrm{I}$ $\mathrm{t}$ is hard to name another phenomenon so commonly encountered as contact electrification ( $\mathrm{CE}$, also known as tribocharging and static electricity), which nevertheless remains so indeterminate when one tries to explain its mechanism. Contact charges develop on surfaces upon contact $^{1-3}$ - even a soft "touch" can give rise to kilovolts of electrical potential on surfaces. In industry (pharmaceuticals, plastics, oil, microelectronics, and space) ${ }^{4-7}$ this electrical potential causes clinging, sparks, friction, and wear, ${ }^{8,9}$ all of which lead to billion-dollar losses. Efforts to prevent these losses by rendering the material surfaces antistatic have aimed at making materials more conductive (e.g., by doping with carbon powder, metals, or conductive polymers) since "all insulators acquire/develop/store surface charges upon contact". However, do all insulators really do that? Since the earliest contact charging experiments millennia ago, ${ }^{10}$ it has been reported that almost all electrically insulating materials can develop charges upon contact. They can be ranked according to this propensity of charge development from negative to positive polarity in a series called the triboelectric series. ${ }^{10-12}$ This ranking is debated even after three centuries since its first report by Wilcke ${ }^{11}$ (1757) because of ambiguous results. $^{10,12}$ This is because contact charging depends on numerous different properties of the material (e.g., surface roughness and crystallinity) $)^{13}$ as well as environmental factors (e.g., humidity and mode of contact), and the mechanism of charging is still unclear. ${ }^{10,14}$ Moreover, identical materials can also be charged, ${ }^{15-18}$ and all materials indeed charge bipolarly in nano-, ${ }^{19}$ micro-, and macrodomains. ${ }^{17,20}$ Further, the measured surface charge is indeed a "net" charge, a mathematical sum of these opposite polarity charges. Nevertheless, in all reported triboelectric series, wood is listed at the center of the series ${ }^{10-12,21}$ as an insulator material that has no tendency for contact charging (Figure 1a). Wood is one of the most commonly used raw materials (annual production of 2 billion metric tons). ${ }^{22}$ It has several advantages over other synthetic alternatives because it does not contribute to environmental pollution, unlike plastics. Wood can serve both as an electrical insulator and antistatic material. Investigating this interesting property of wood is more than just a fundamental research interest; it is a necessity for the development of current and new wood technologies. ${ }^{23}$ One example of the utilization of treated wood is recently shown in the inventive work by Luo et al. in flexible and durable triboelectric generators in self-powered sensors. ${ }^{24}$

\section{WOOD IS "SUPER-ANTISTATIC" UNLESS LIGNIN IS REMOVED FROM IT}

The inertness of wood to contact charging is compared to the contact charging behaviors of two common polymers, polydimethylsiloxane (PDMS) and nylon, in Figure $1 \mathrm{~b}$. When PDMS and nylon were touched against aluminum foil,

Received: June 9, 2020

Revised: August 7, 2020

Published: August 11, 2020 
a)

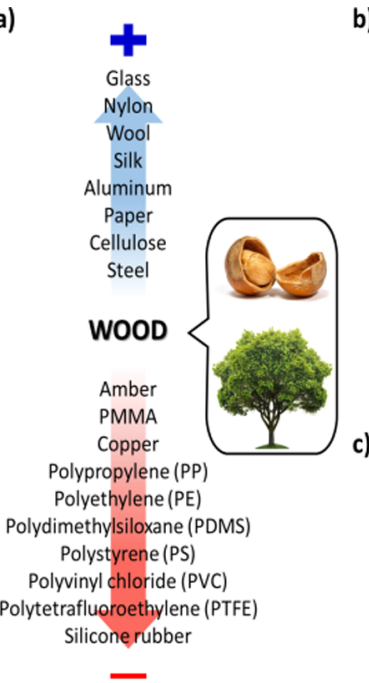

b)

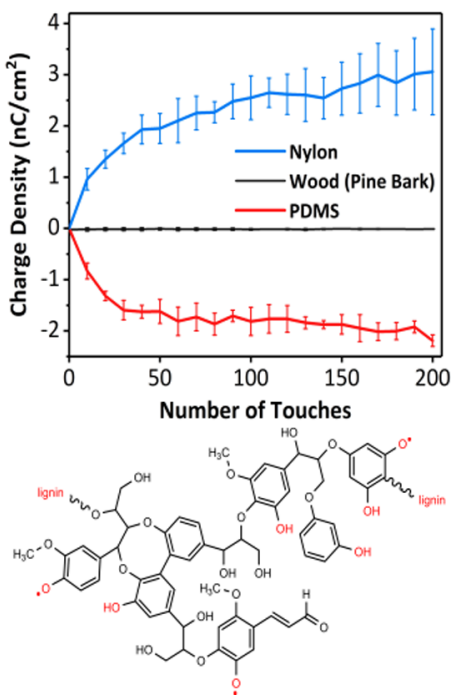

Figure 1. (a) Always placed at the center of triboelectric series, wood is both an insulating and a non-CE material. (b) CE behavior of wood $\left(Q=\sim 20 \mathrm{pC} / \mathrm{cm}^{2}\right)$ in comparison to that of PDMS and nylon. All materials were touched against $\mathrm{Al}$ foil. (c) Typical chemical structure of lignin. Error bars correspond to standard deviations determined from at least four independent experiments. See the Supporting Information for further experimental details on sample preparation and charging experiments.

after several touches, they acquired ca. -2.0 and $+2.7 \mathrm{nC} / \mathrm{cm}^{2}$ net surface charge, respectively, while a typical wood sample did not charge at all (having a charge density less than $20 \mathrm{pC} /$ $\mathrm{cm}^{2}$ ) even after 200 touches. Here, we propose that this natural "super-antistatic" action of wood can be attributed to the

presence of high amounts of lignin in its structure (vide infra and Figure 1c) and that this property can be used to make common polymers antistatic by doping them with only minute amounts of lignin, the second most abundant polymer on Earth.

To understand the inertness of wood against contact charging, it is necessary to understand how and why common polymers get charged at all. As mentioned above, this is not an easy task because of the complicated nature of contact charging. Previously, it was shown that electron, ion, and material transfer play important roles during the $\mathrm{CE}$ process. $^{25-30}$ More recently, we $\mathrm{e}^{19,31,32}$ and others ${ }^{20,33-36}$ proved a chemical mechanism wherein polymer bonds break to yield mechano-ions, ${ }^{34-40}$ which are indeed the observed contact charges. This chemical mechanism also reveals the role of mechanoradicals in $\mathrm{CE}$, i.e., in stabilizing the charges (mechano-ions) on the surfaces. ${ }^{32}$ The inertness of wood against CE that persists for all kinds of wood with different physical features such as roughness and fiber morphology might as well be explained by this mechanism, if one considers the chemical constituents of the wood that are common to all wood. Wood is a composite of three natural polymers, namely, cellulose $(30-50 \%)$, hemicellulose $(20-35 \%)$, and lignin $(15-40 \%) .{ }^{23,41}$ The intervention of the minor ingredients of wood, e.g., organic compounds, was eliminated by washing the wood samples with organic solvents and drying them prior to the experiments (these washed samples displayed identical charging behavior as the native ones). As it is known that cellulose breaks mechanically ${ }^{35,42-44}$ and acquires contact charges, and that hemicellulose has the same chemistry as cellulose, we suspected that lignin was responsible for the observed "super-antistatic" action.

a)

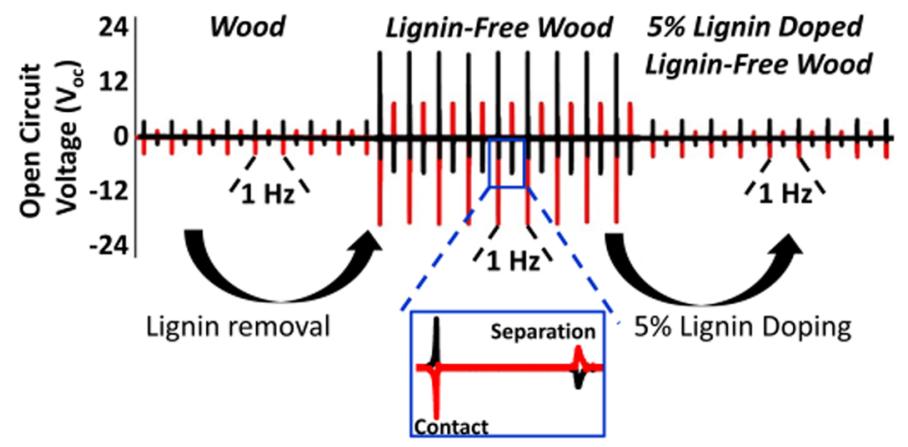

b)
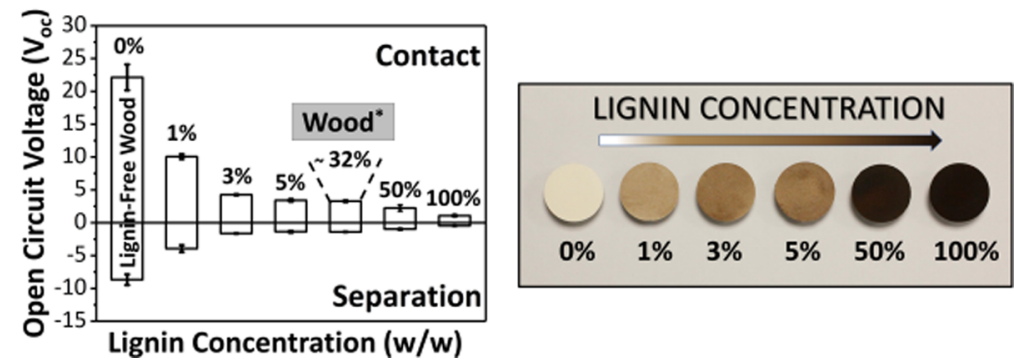

Figure 2. (a) Removing lignin from wood (here, limba wood) precludes its non-CE behavior, and doping the lignin-free wood (LFW) with 5\% lignin restores it. Wood, LFW, and lignin-doped wood are contact charged at a tapping device against a polytetrafluoroethylene (PTFE) film at 1 $\mathrm{Hz}$ frequency. (Inset) Contact and separation open-circuit potential $\left(V_{\mathrm{oc}}\right)$ signals from wood samples (red) and PTFE (black). Even small amounts of lignin in wood (1\%) decrease the $V_{\text {oc }}$ (b) $V_{\text {oc }}$ obtained using natural wood (*limba, 32\% lignin, sample prepared in an identical manner as the lignin-free samples via cryomilling and pressing into a pellet) in comparison to artificially doped LFW samples. Error bars correspond to standard deviations determined from at least four independent experiments. See the Supporting Information for further experimental details on sample preparation and contact charging experiments. 
a)

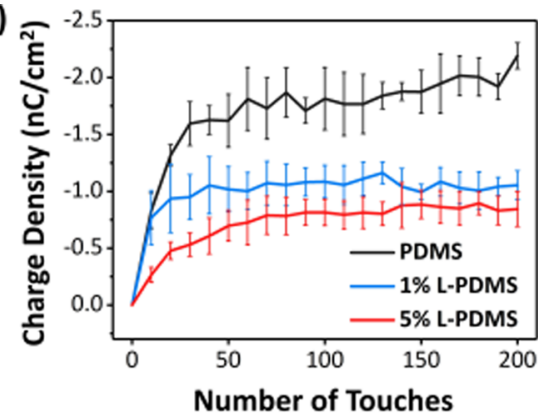

c)

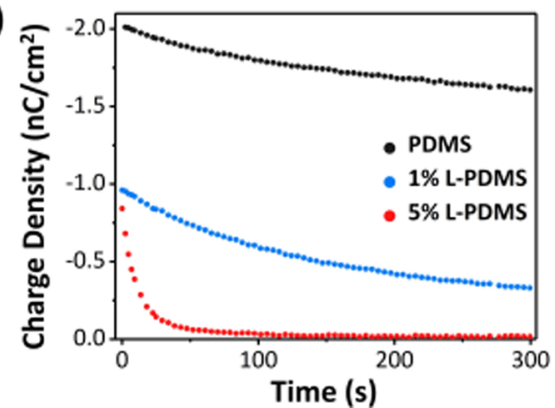

b)

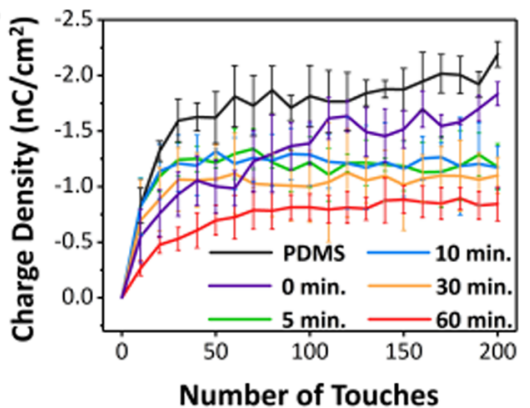

d)

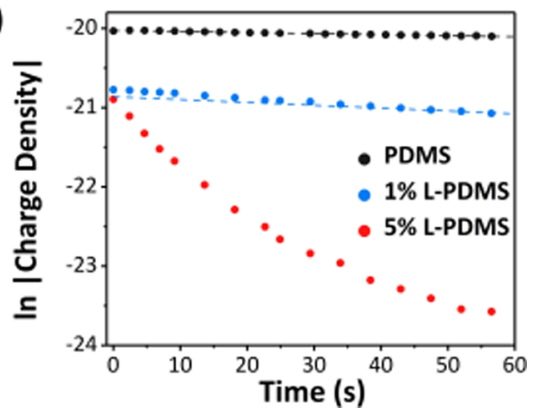

Figure 3. (a) Contact charge density of PDMS reduces upon lignin doping (L-PDMS). (b) Increasing the cryomilling time of lignin before doping ensures finer particles and better dispersion of lignin in PDMS (5\% (w/w) doping). (c) Contact charges in panel (a) decay faster on PDMS as more lignin is added (L-PMDS, w/w). (d) Charge decay rates in panel (c) show deviations from linearity (from black to red plots) upon lignin doping to PDMS. Error bars correspond to standard deviations determined from at least four independent experiments. See the Supporting Information for further experimental details.

To test the hypothesis that wood free of lignin would develop contact charge same as cotton or synthetic polymers, we removed lignin from wood by chemical extraction ${ }^{45}$ and prepared a pellet $(1.4 \mathrm{~cm}$ diameter and $0.5 \mathrm{~mm}$ thick, Figure 2 ) from the remaining cellulosic material by cryomilling the samples and pressing the obtained powder. (See the Supporting Information (SI) for further experimental details on extraction and sample preparation, Figures S1-S4).

To display the charging behavior of lignin-free wood (LFW), we used a tapping device set at a tapping frequency of $1 \mathrm{~Hz}$, which allowed the contact and separation of the wood samples with $\mathrm{Al}$ metal. The tapping device had two identical metal stubs (Al) attached directly to $100 \mathrm{M} \Omega$ (high enough input impedance $^{46}$ to prevent discharge of the signals during tapping) oscilloscope probes, one of which was mounted on the wood sample. The CE of surfaces during their contact and separation led to open-circuit electrical potential ( $V_{\text {oc }}$ in volts) of opposite signs, which were measured independently using a two-channel oscilloscope. By using a tapping device, mechanical inconsistencies that emerge from pressure differences upon manual contact were eliminated. Qualitatively, experiments by manual contact, which can be performed in a straightforward manner, gave similar results. For details about the experimental parameters, see the Supporting Information. As shown in Figure 2a, the LFW sample produced a high $V_{\text {oc }}$ of $\sim 22 \mathrm{~V}$ (similar to common synthetic polymers producing 6$16 \mathrm{~V}$ (Figure 5)), which was about an order of magnitude higher than that can be obtained by native wood samples; e.g., for limba wood containing $\sim 32 \%$ lignin, ${ }^{47} V_{\text {oc }}$ was determined to be $\sim 3.2 \mathrm{~V}$ on the same device. (A complete removal of lignin is needed for charging; the samples with partial lignin removal were found to be resistant to charging, Figure S4). To probe the effect of lignin concentration in wood to tribocharging, we doped LFW with $1-50 \%$ lignin. Figure $2 b$ clearly shows that the $V_{\text {oc }}$ dropped rapidly upon addition of lignin in the wood-even at $1 \%$ of doping, the $\mathrm{V}_{\mathrm{oc}}$ decreased by half of that obtained with LFW ( $0 \%$ lignin), illustrating the "super-antistatic" action of lignin.

Next, to test the antistatic action of lignin in a systematic manner and uncover details of the charge dissipation mechanism of lignin, we doped lignin (see the Supporting Information, Figures $\mathrm{S} 1-\mathrm{S} 12$ for further experimental details and extraction and characterization of the lignin samples) extracted from different sources (birch, pine, maple tree barks, and nutshell, containing $30-40 \%(\mathrm{w} / \mathrm{w}) \operatorname{lignin}^{48}$ extracted through the Klason process; see the Supporting Information) into polydimethylsiloxane (PDMS, Dow Sylgard 184), a common polymer used in CE studies. As shown in Figure $3 a$, even at $1 \%$ lignin dopant concentration, the charge accumulation on the lignin-doped PDMS (L-PDMS) surface decreased by $52 \%$, and this decrease was enhanced if lignin was cryomilled into fine particles and well dispersed in the polymer matrix (Figure 3b and Figure S12). As shown in AFM images of the doped and undoped PDMS samples (Figure S13), the surface roughness increases upon doping with lignin. On contrary to the common trend showing an increase in charging of polymers with increasing roughness, ${ }^{49}$ the charge densities of the rougher lignin-doped samples were found to be less than undoped ones, showing that the reduction in charge density is not because of the increase in surface roughness. This trend was shown to be independent of the counter material $(\mathrm{Cu}, \mathrm{Al}$, PTFE, and steel, as shown in Figure S14) and also of the lignin source (Figure S15). As seen from the discharge plots (Figure $3 \mathrm{c}$ ) of the undoped and doped PDMS, the antistatic action was also reflected in the faster charge decay observed with the latter (rate constant of $2.31 \times 10^{-3} \mathrm{~s}^{-1}$ for $1 \%$ doping) in comparison to undoped PDMS (rate constant of $1.32 \times$ $10^{-3} \mathrm{~s}^{-1}$ ) (Figure 3d). The decay rate deviates from first order 
a)

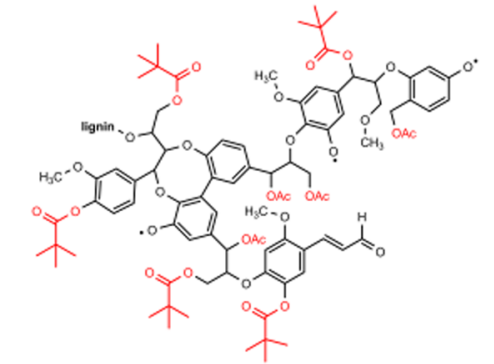

c)

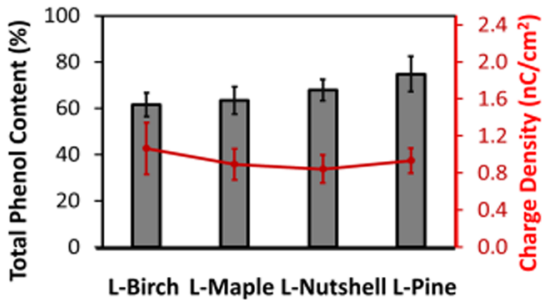

b)
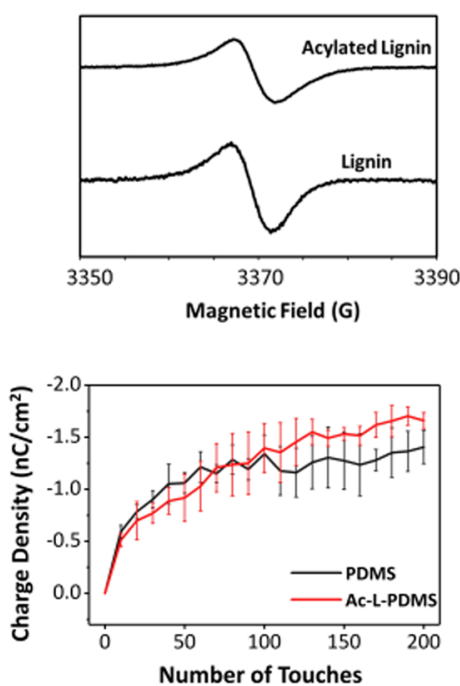

Figure 4. (a) Chemical structure of acylated lignin (Ac: trimethyl acetyl). (b) Normalized electron spin resonance (ESR) spectra of lignin and acylated lignin showing both have phenoxy radicals (g value: 2.003). (c) Lignins extracted from different sources with similar total phenol content dissipate contact charges similarly when doped into PDMS. (d) Surface charge density of undoped PDMS and acylated lignin-doped PDMS (Ac-LPDMS $)(5 \% \mathrm{w} / \mathrm{w})$ when both were touched against $\mathrm{Al}$ foil. The plot shows that the removal of active phenolic $\mathrm{H}$ (substituted by acyl groups) inhibits the antistatic action of lignin. $\mathrm{RH}=20 \%$. Error bars correspond to standard deviations determined from at least four independent experiments. See the Supporting Information for further experimental details on sample preparation and CE experiments.

upon doping, implying that a second species is involved in the decay mechanism. ${ }^{50,5}$

\section{CHEMICAL MECHANISM OF ANTISTATIC ACTION OF LIGNIN}

The remarkable antistatic action of lignin is interesting because lignin is not inherently conductive like conventional antistatic additives such as metal particles or synthetic conductive polymers. To show that the presence of lignin does not increase the surface conductivities of the samples, we measured the conductivities of the corresponding samples by the twoprobe method (see the Supporting Information for experimental details). The surface conductivity of the 5\% L-PDMS sample was found to be of the same order as that of undoped PDMS ( $\sim 10^{-15} \mathrm{~S} / \mathrm{m}$, typical of an insulator) (Figure S19). Another charge dissipation mechanism of common antistatic additives, in which water (that eliminates surface charges by percolation) absorption is enhanced on the polymer surfaces by the additives, was also refuted by the observation that surface humidity did not increase upon addition of lignin into LFW (Figure S11). Based on these observations, we sought an explanation for the antistatic action of lignin in the bondbreaking mechanism of contact charging. This mechanism $^{19,20,28,30-34,52}$ states that the mechanical action (contact) breaks the chemical bonds on the surface of the polymers leading to the formation of mechano-ions (charges) and mechanoradicals. The contact induced bond breaking in polymers upon polymer-polymer and polymer-metal contact that can be evidenced by the material transfer, ${ }^{28-30}$ which can be verified by X-ray photoelectron spectroscopy (XPS). ${ }^{19,28}$ For the samples used in the charging experiments in this study (Figure S14), XPS analyses show such a material transfer and bond breaking on polymer surfaces (Figure S16). AFM/KFM was also employed to image the distribution of surface charge before and after contact (Figure S13). The KFM images of PDMS and L-PDMS samples are qualitatively similar, i.e., they both show that the charging is heterogeneous (both positive and negative), and the charge domains are mostly concentrated at the locations with increased roughness (bond breaking). So, one can conclude that the contact-charging is a result of bond breaking in both cases. Once the bonds are broken and mechanospecies are formed, mechanoradicals can stabilize these charges, and their removal with a radical scavenger destabilizes the charges, rendering the material antistatic. ${ }^{32,33}$

In lignin, this radical scavenger can either be (a) the stable radicals in lignin (also responsible for its known antioxidant activity) ${ }^{53}$ that can react with the polymer mechanoradicals (in wood, these are the cellulose mechanoradicals ${ }^{42-44}$ ) that form upon CE of the polymer (or wood) or (b) phenols in the lignin structure that can form more stable radicals upon reaction with these polymer (or wood) mechanoradicals by $\mathrm{H}$ atom transfer. In the second mechanism, the formed phenolic stable radicals cannot interact with the charges (polymer mechano-ions) to stabilize them because of their nonmatching energy levels, ${ }^{54}$ which makes charge dissipation faster. These mechanisms are summarized in Figure S17. We surmise that the second mechanism predominates because the number of lignin mechanoradicals (on the order of $\left.\sim 10^{18} / \mathrm{g}\right)^{53,55}$ is quite low in comparison to the number of phenols $(3.3-4.0 \mathrm{mmol} /$ g, Table S1). This hypothesis was further supported by the observation that the PDMS sample doped with acylated lignin (prepared following a similar procedure as for L-PDMS), which lacks the $\mathrm{OH}$ groups for the required $\mathrm{H}$-atom transfer but possesses lignin radicals (Figure 4 and Figure S17), did not show any antistatic action and charged identically as undoped PDMS. The antistatic action of lignin was found to be quantitatively similar in different types of wood (birch, pine, maple, and nutshell) (Figure S15), for which the phenolic content and chemical nature of the phenols were found to be similar through ${ }^{13} \mathrm{C}-\mathrm{NMR}$ analyses (Figure S9). 


\section{UNIVERSALITY OF ANTISTATIC ACTION OF LIGNIN IN COMMON POLYMERS}

Inspired by the successful antistatic action of lignin in PDMS, we attempted to dope several other polymers with lignin to show the universality of this action. We aimed to make common polymers antistatic with lignin, probably the most abundant and cheapest polymeric material that can exist for this action. For this purpose, samples from some common thermoplastic polymers, such as polypropylene (PP), polylactic acid (PLA), polyethylene (PE), and polystyrene (PS), were prepared $(1.2 \mathrm{~cm}$ diameter and $0.4 \mathrm{~mm}$ thick) and mounted on one of the stubs connected to the probe of the oscilloscope. Using the tapping instrument, the samples were tapped on the $\mathrm{Al}$ stub connected to the other probe, and $V_{\mathrm{oc}}$ values were recorded (Figure 5). It was observed that 5\% (w/w) lignin

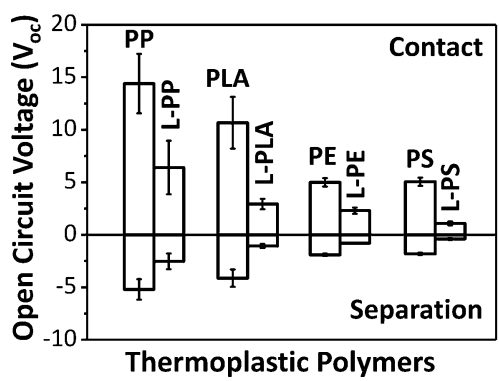

Figure 5. CE propensity of the thermoplastic polymers (measured as open-circuit voltage on a tapping setup) before and after 5\% lignin doping. The open-circuit voltages are saturated signals collected from the oscilloscope. See the Supporting Information for further experimental details. Error bars correspond to standard deviations determined from at least four independent experiments.

doping in the samples indeed decreased charge accumulation by $60-80 \%$, as detected from the saturated signals of $V_{\text {oc }}$ of the polymer and metal, both upon contact and separation. ${ }^{46}$

With this study, we have provided insight to the chemical mechanism of why wood does not get contact charged, showing the antistatic action of lignin, a naturally occurring, low-cost material. ${ }^{41}$ Our results also demonstrate that lignin might be a good antistatic additive for common polymers even at low doping concentrations to prevent charge accumulationbased hazards in industry. Further, our explanation of the antistatic action of lignin in contact-charged polymers might prove helpful in understanding the millennia-long debated molecular mechanism of contact charging and charge dissipation in common polymers. Finally, our findings illustrate a long-awaited sustainable technological solution to $\mathrm{CE}$ by employing one of the most abundant natural materials, wood.

\section{EXPERIMENTAL SECTION}

Materials. The nutshell was obtained from nuts grown in Gümeli village in the west black sea region, Turkey. Maple tree bark, pine tree bark, and birch tree bark were collected from their natural habitat in Bilkent University Campus, Ankara, Turkey. Sulfuric acid (95-97\%), acetic acid (99.85\%, glacial), phosphoric acid (85\%), hydrochloric acid (37\%), 2,2-diphenyl-1-picrylhydrazyl (DPPH), and thermoplastics (PP, PE, PS, and PLA) were purchased from Sigma Aldrich. Tetrahydrofuran, ethanol, isopropyl alcohol, 1,4-dioxane, and acetonitrile from Sigma Aldrich were used without further purification. $\mathrm{H}_{2} \mathrm{O}_{2}$ solution, $\mathrm{FeCl}_{3}, \mathrm{~K}_{3} \mathrm{Fe}(\mathrm{CN})_{6}$, trimethylacetyl chloride, and methyl-3,4,5-trihydroxybenzoate (Sigma Aldrich) were used as obtained. $\mathrm{NaOH}$ (pellets) and $\mathrm{Na}_{2} \mathrm{SO}_{3}$ were purchased from Carlo Erba. Arabic gum was purchased from an herbalist. PDMS was prepared by using a Dow Corning Sylgard 184 silicone elastomer kit. The samples for the electrical potential measurements using the tapping device described below were mounted on aluminum stubs from Agar Scientific.

Instrumentation. For the lignin extraction, an autoclave reactor $(30 \mathrm{~mL})$ (PARR Instrument Company) was used. Retsch molecular test sieves (50 and 100 micron mesh size) and Retsch cryomill were used to decrease the particle size of the samples. The samples were placed in a $50 \mathrm{~mL}$ zirconia grinding chamber and cryomilled with six zirconia balls (diameter: $10.06 \mathrm{~mm}$ ) at $30 \mathrm{~Hz}$ frequency at $77 \mathrm{~K}$. Particle size measurements were performed on a Malvern instrument zeta-sizer. FTIR-ATR spectra were taken using a Bruker Alpha FTIRATR spectrometer. All samples were analyzed with a spectral width of $4.000-400 \mathrm{~cm}^{-1}$ and 64 scans at a resolution of $4 \mathrm{~cm}^{-1}$. The spectra were baseline corrected, and transmittance was normalized. Electron spin resonance (ESR) analyses were performed for noncryomilled lignin, cryomilled lignin, and acylated lignin. Mechanochemically generated radicals were characterized at room temperature under nonsaturating conditions using a Bruker ELEXYSY E 580 model ESR spectrometer equipped with a high sensitivity cavity and operating at $\mathrm{X}$ band frequencies $(9 \mathrm{GHz})$. ESR signals were obtained by double integration of the corrected baseline using Bruker Win EPR software. Experimental conditions were set to $12 \mathrm{~dB}, 1 \mathrm{G}, 0.3 \mathrm{~mW}$ microwave power, $0.25 \mathrm{mT}$ modulation amplitude, and 1024 points. The molecular weights of the lignin samples were determined by an SEC system (Agilent, Santa Clara, CA, USA) with a diode array UV detector (Agilent 1200 series ELSD), and the mobile phase was THF (HPLC grade, without stabilizer) with a flow rate of $0.6 \mathrm{~mL} / \mathrm{min}$. The column used was $6.2 \mathrm{~mm} \times 250 \mathrm{~mm}$ Agilent Zorbax PSM 300-S (particle size: $5 \mu \mathrm{m}$ ). The system was calibrated with polystyrene standards $(575,1530,3950,10,210,29,510,72,450,205,000$, and 467,000 Da) using an ELSD detector. ${ }^{13} \mathrm{C}-\mathrm{CP} / \mathrm{MAS}$ NMR analyses were carried out using a Bruker Avance $300 \mathrm{MHz}$ WB superconducting FT NMR spectrometer equipped with a $4 \mathrm{~mm}$ MAS probe. For acquisition of the ${ }^{13} \mathrm{C}$-CP/MAS NMR spectra, a relaxation delay of $4 \mathrm{~s}$ and spin rate of $8.5 \mathrm{kHz}$ were used. Then, 7000 scans were taken for each spectrum. The NMR spectrometer was calibrated against a pure glycine sample before the measurements. XPS spectra were recorded on ESCALAB 250 (Thermo Scientific K-Alpha X-ray photoelectron spectrometer). Photoemission was stimulated by a monochromatic $\mathrm{Al} \mathrm{K} \alpha$ radiation $(1486.6 \mathrm{eV})$. Survey scans and highresolution scans were collected using pass energies of 200 and $30 \mathrm{eV}$, respectively. Binding energies in the spectra were referenced to the $\mathrm{C}$ $1 \mathrm{~s}$ binding energy set at $284.8 \mathrm{eV}$. At least three different measurements were performed for each sample. For AFM/KFM imaging, a Nanosurf AFM microscope was used.

Charge Density and Open-Circuit Potential Measurements. Electrostatic charges on the polymer surfaces were measured by immersing the polymer pieces in a homemade Faraday cup attached to an electrometer (Keithley 6517B). Lignin-doped and undoped PDMS samples were contact charged against aluminum foil up to 200 touches (Figure S18a). Since it is harder to manipulate hard plastics with tweezers during this process, charging behaviors of thermoplastics were monitored using a separate method that utilizes a homemade tapping device attached to an oscilloscope (Figure S18b). In this method, surface electrical potential of thermoplastics that increases upon contact charging is recorded as the signal, opencircuit potential $V_{\text {oc }}$. Samples were mounted on the aluminum stubs that are connected to the electrodes of the electrometer. Open- circuit voltages were measured and collected from saturated signals (signals obtained when accumulated charges are at their maximum values). A $1 \mathrm{~Hz}$ tapping frequency was used. For some samples, due to low propensity for charging on $\mathrm{Al}$, a PTFE (Teflon)-coated stub surface was also used as the counter electrode. Standard deviations were calculated from at least four independent measurements. In case otherwise is stated, $\mathrm{RH}=23-28 \%$.

Charge Decay Measurements. Before charge decay experiments, PDMS pieces were left to discharge for at least $24 \mathrm{~h}$ in an isolated container. The electroneutrality of these pieces was confirmed by immersing the pieces in a home-made Faraday cup connected to a 
high-precision electrometer (Keithley Instruments, model 6517B) that measures electrical charge. Undoped and doped PDMS pieces were charged against aluminum foil several times in order to reach the highest surface charge (charge saturation point). Then, samples were kept immersed in the homemade Faraday cup up to $30 \mathrm{~min}$. Charge decay rates were calculated using OriginPro by linear equation fitting.

Surface Conductivity Measurements. In order to investigate whether the fast decay in the case of lignin doping is caused by the increase in surface conductivity of doped pieces, surface conductivities of PDMS and 5\% lignin-doped PDMS samples were measured using a two-probe method. Current versus voltage curves of undoped PDMS and 5\% lignin-doped PDMS was obtained via a probe station (with $w$ $=1 \mathrm{~cm}$ wide samples $(1 \mathrm{~cm} \times 1 \mathrm{~cm}$ square pieces), and the distance between copper electrodes $d=100 \mu \mathrm{m}$ ), and the applied voltage was changed from 0 to -100 and 0 to $+100 \mathrm{~V}$ in steps of $10 \mathrm{~V}$, which gave identical results in terms of surface conductivity. From the slopes of the $I-V$ curves, the values for surface resistance $R_{\mathrm{s}}$ were calculated according to the equation $R_{s}=(V / I) \cdot(w / d)$ in $\Omega /$ sq. Then, surface resistivities were converted to surface conductivity $(\sigma)$ using the equation $\sigma=1 / R_{\mathrm{s}}$. Standard deviations of the surface conductivities were calculated from at least four independent measurements.

\section{ASSOCIATED CONTENT}

\section{SI Supporting Information}

The Supporting Information is available free of charge at https://pubs.acs.org/doi/10.1021/acs.chemmater.0c02421.

Experimental procedures of lignin extraction, lignin acylation, and lignin doping to polymers; detailed characterization of the samples $\left({ }^{13} \mathrm{C}-\mathrm{NMR}\right.$, TGA/DSC, AFM/KFM, XPS, ESR, GPC, and total phenol content analyses); and proposed chemical mechanisms and electrical measurements (PDF).

\section{AUTHOR INFORMATION}

\section{Corresponding Author}

Bilge Baytekin - Department of Chemistry and UNAM National Nanotechnology Research Center, Bilkent University, Ankara 06800, Turkey; ○ orcid.org/0000-0002-3867-3863; Email: b-baytekin@fen.bilkent.edu.tr

\section{Authors}

Mertcan Özel - Department of Chemistry, Bilkent University, Ankara 06800, Turkey

Fatma Demir - Department of Chemistry, Bilkent University, Ankara 06800, Turkey

Aizimaiti Aikebaier - UNAM National Nanotechnology Research Center, Bilkent University, Ankara 06800, Turkey

Joanna Kwiczak-Yiğitbaşı - Department of Chemistry, Bilkent University, Ankara 06800, Turkey

H. Tarik Baytekin - Department of Chemistry, Bilkent University, Ankara 06800, Turkey

Complete contact information is available at:

https://pubs.acs.org/10.1021/acs.chemmater.0c02421

\section{Author Contributions}

${ }^{\S}$ M.O. and F.D. contributed equally.

\section{Notes}

The authors declare no competing financial interest.

\section{ACKNOWLEDGMENTS}

We thank the Scientific and Technological Research Council of Turkey (TÜBITTAK) (project no. 116Z523) for financial support, METU Central Laboratory, and Dr. Sedat Canlı for electron spin resonance (ESR) measurements.

\section{REFERENCES}

(1) Horn, R. G.; Smith, D. T.; Grabbe, A. Contact Electrification Induced by Monolayer Modification of a Surface and Relation to Acid-Base Interactions. Nature 1993, 366, 442-443.

(2) Horn, R. G.; Smith, D. T. Contact Electrification and Adhesion between Dissimilar Materials. Science 1992, 256, 362-364.

(3) Coehn, A. Ueber Ein Gesetz Der Electricitätserregung. Ann. Phys. 1898, 300, 217-232.

(4) Angelopoulos, M. Conducting Polymers in Microelectronics. IBM J. Res. Dev. 2001, 45, 57-75.

(5) Gibson, N. Static Electricity - An Industrial Hazard under Control? J. Electrost. 1997, 40-41, 21-30.

(6) Greason, W. D. Review of the Effect of Electrostatic Discharge and Protection Techniques for Electronic Systems. IEEE Trans. Ind. Appl. 1987, IA-23, 205-216.

(7) Lai, S. T. Spacecraft Charging: Progress in Astronautics and Aeronautics; Vol. 237, American Institute of Aeronautics and Astronautics: Reston, VA, 2011.

(8) Sayfidinov, K.; Cezan, S. D.; Baytekin, B.; Baytekin, H. T. Minimizing Friction, Wear, and Energy Losses by Eliminating Contact Charging. Sci. Adv 2018, 4, No. eaau3808.

(9) Galembeck, F.; Burgo, T. A. L.; Balestrin, L. B. S.; Gouveia, R. F.; Silva, C. A.; Galembeck, A. Friction, Tribochemistry and Triboelectricity: Recent Progress and Perspectives. RSC Adv. 2014, 4, 64280-64298.

(10) Lacks, D. J.; Shinbrot, T. Long-Standing and Unresolved Issues in Triboelectric Charging. Nat. Rev. Chem. 2019, 3, 465-476.

(11) Wilcke, J. C. Dispvtatio Physica Experimentalis, de Electricitatibvs Contrarii [Latin]; Typis Joannis Jacobi Adleri: Rostochii, 1757.

(12) Diaz, A. F.; Felix-Navarro, R. M. A Semi-Quantitative TriboElectric Series for Polymeric Materials: The Influence of Chemical Structure and Properties. J. Electrost. 2004, 62, 277-290.

(13) Wang, A. E.; Gil, P. S.; Holonga, M.; Yavuz, Z.; Baytekin, H. T.; Sankaran, R. M.; Lacks, D. J. Dependence of Triboelectric Charging Behavior on Material Microstructure. Phys. Rev. Mater. 2017, 1, No. 035605.

(14) Williams, M. W. Triboelectric Charging of Insulating PolymersSome New Perspectives. AIP Adv. 2012, 2, No. 010701.

(15) Apodaca, M. M.; Wesson, P. J.; Bishop, K. J. M.; Ratner, M. A.; Grzybowski, B. A. Contact Electrification between Identical Materials. Angew. Chem., Int. Ed. 2010, 49, 946-949.

(16) Lowell, J.; Truscott, W. S. Triboelectrification of Identical Insulators. I. An Experimental Investigation. J. Phys. D: Appl. Phys. 1986, 19, 1273-1280.

(17) Shinbrot, T.; Komatsu, T. S.; Zhao, Q. Spontaneous Tribocharging of Similar Materials. Europhys. Lett. 2008, 83, 24004.

(18) Shaw, P. E. Electrical Separation Between Identical Solid Surfaces. Proc. Phys. Soc. 1926, 39, 449-452.

(19) Baytekin, H. T.; Patashinski, A. Z.; Branicki, M.; Baytekin, B.; Soh, S.; Grzybowski, B. A. The Mosaic of Surface Charge in Contact Electrification. Science 2011, 333, 308-312.

(20) Burgo, T. A. L.; Ducati, T. R. D.; Francisco, K. R.; Clinckspoor, K. J.; Galembeck, F.; Galembeck, S. E. Triboelectricity: Macroscopic Charge Patterns Formed by Self-Arraying Ions on Polymer Surfaces. Langmuir 2012, 28, 7407-7416.

(21) Zou, H.; Zhang, Y.; Guo, L.; Wang, P.; He, X.; Dai, G.; Zheng, H.; Chen, C.; Wang, A. C.; Xu, C.; Wang, Z. L. Quantifying the Triboelectric Series. Nat. Commun. 2019, 10, 1427.

(22) OECD report, Materials, Resources, Productivity, and The Environment; 2013; pp. 4.

(23) Zhu, H.; Luo, W.; Ciesielski, P. N.; Fang, Z.; Zhu, J. Y.; Henriksson, G.; Himmel, M. E.; Hu, L. Wood-Derived Materials for Green Electronics, Biological Devices, and Energy Applications. Chem. Rev. 2016, 116, 9305-9374.

(24) Luo, J.; Wang, Z.; Xu, L.; Wang, A. C.; Han, K.; Jiang, T.; Lai, Q.; Bai, Y.; Tang, W.; Fan, F. R.; Wang, Z. L. Flexible and Durable Wood-Based Triboelectric Nanogenerators for Self-Powered Sensing in Athletic Big Data Analytics. Nat. Commun. 2019, 10, 5147. 
(25) Liu, C.; Bard, A. J. Electrostatic Electrochemistry at Insulators. Nat. Mater. 2008, 7, 505-509.

(26) McCarty, L. S.; Winkleman, A.; Whitesides, G. M. Ionic Electrets: Electrostatic Charging of Surfaces by Transferring Mobile Ions upon Contact. J. Am. Chem. Soc. 2007, 129, 4075-4088.

(27) McCarty, L. S.; Whitesides, G. M. Electrostatic Charging Due to Separation of Ions at Interfaces: Contact Electrification of Ionic Electrets. Angew. Chem., Int. Ed. 2008, 47, 2188-2207.

(28) Baytekin, H. T.; Baytekin, B.; Incorvati, J. T.; Grzybowski, B. A. Material Transfer and Polarity Reversal in Contact Charging. Angew. Chem., Int. Ed. 2012, 51, 4843-4847.

(29) Fan, F. R.; Luo, J.; Tang, W.; Li, C.; Zhang, C.; Tian, Z.; Wang, Z. L. Highly Transparent and Flexible Triboelectric Nanogenerators: Performance Improvements and Fundamental Mechanisms. J. Mater. Chem. A 2014, 2, 13219-13225.

(30) Pandey, R. K.; Kakehashi, H.; Nakanishi, H.; Soh, S. Correlating Material Transfer and Charge Transfer in Contact Electrification. J. Phys. Chem. C 2018, 122, 16154-16160.

(31) Baytekin, B.; Baytekin, H. T.; Grzybowski, B. A. What Really Drives Chemical Reactions on Contact Charged Surfaces? J. Am. Chem. Soc. 2012, 134, 7223-7226.

(32) Baytekin, H. T.; Baytekin, B.; Hermans, T. M.; Kowalczyk, B.; Grzybowski, B. A. Control of Surface Charges by Radicals as a Principle of Antistatic Polymers Protecting Electronic Circuitry. Science 2013, 341, 1368-1371.

(33) Fang, Y.; Gonuguntla, S.; Soh, S. Universal Nature-Inspired Coatings for Preparing Noncharging Surfaces. ACS Appl. Mater. Interfaces 2017, 9, 32220-32226.

(34) Sakaguchi, M.; Shimada, S.; Kashiwabara, H. Mechanoions Produced by Mechanical Fracture of Solid Polymer. 6. A Generation Mechanism of Triboelectricity Due to the Reaction of Mechanoradicals with Mechanoanions on the Friction Surface. Macromolecules 1990, 23, 5038-5040.

(35) Sakaguchi, M.; Makino, M.; Ohura, T.; Iwata, T. Contact Electrification of Polymers Due to Electron Transfer among Mechano Anions, Mechano Cations and Mechano Radicals. J. Electrost. 2014, 72, 412-416.

(36) Galembeck, F.; Burgo, T. A. L. Chemical Electrostatics: New Ideas on Electrostatic Charging: Mechanisms and Consequences; Springer: Cham, 2017.

(37) Baytekin, B.; Baytekin, H. T.; Grzybowski, B. A. Mechanically Driven Activation of Polyaniline into Its Conductive Form. Angew. Chem., Int. Ed. 2014, 53, 6946-6950.

(38) Urbanski, T. Degradation of Amber and Formation of Free Radicals by Mechanical Action. Proc. R. Soc. London, Ser. A 1971, 325, 377-381.

(39) Beyer, M. K.; Clausen-Schaumann, H. Mechanochemistry: The Mechanical Activation of Covalent Bonds. Chem. Rev. 2005, 105, 2921-2948.

(40) Sohma, J. Mechano-Radical Formation in Polypropylene by an Extruder Action and Its after-Effects. Colloid Polym. Sci. 1992, 270, $1060-1065$.

(41) Upton, B. M.; Kasko, A. M. Strategies for the Conversion of Lignin to High-Value Polymeric Materials: Review and Perspective. Chem. Rev. 2016, 116, 2275-2306.

(42) Laçin, Ö.; Kwiczak-Yiğitbaşı, J.; Erkan, M.; Cevher, Ş. C.; Baytekin, B. The Morphological Changes upon Cryomilling of Cellulose and Concurrent Generation of Mechanoradicals. Polym. Degrad. Stab. 2019, 168, 108945.

(43) Sakaguchi, M.; Ohura, T.; Iwata, T.; Enomoto-Rogers, Y. Nano Cellulose Particles Covered with Block Copolymer of Cellulose and Methyl Methacrylate Produced by Solid Mechano Chemical Polymerization. Polym. Degrad. Stab. 2012, 97, 257-263.

(44) Kwiczak-Yiğitbaşi, J.; Laçin, Ö.; Demir, M.; Ahan, R. E.; Şeker, U. Ö. Ş.; Baytekin, B. A Sustainable Preparation of Catalytically Active and Antibacterial Cellulose Metal Nanocomposites: Via Ball Milling of Cellulose. Green Chem. 2020, 22, 455-464.
(45) Zhu, M.; Song, J.; Li, T.; Gong, A.; Wang, Y.; Dai, J.; Yao, Y.; Luo, W.; Henderson, D.; Hu, L. Highly Anisotropic, Highly Transparent Wood Composites. Adv. Mater. 2016, 28, 5181-5187.

(46) Musa, U. G.; Cezan, S. D.; Baytekin, B.; Baytekin, H. T. The Charging Events in Contact-Separation Electrification. Sci. Rep. 2018, 8,2472 .

(47) Zobel, B. J.; van Buijtenen, J. P. Wood Variation: Its Causes and Control; Springer Science \& Business Media: 2012; pp. 69.

(48) Ponnusamy, V. K.; Nguyen, D. D.; Dharmaraja, J.; Shobana, S.; Banu, J. R.; Saratale, R. G.; Chang, S. W.; Kumar, G. A Review on Lignin Structure, Pretreatments, Fermentation Reactions and Biorefinery Potential. Bioresour. Technol. 2019, 271, 462-472.

(49) Neagoe, M. B.; Prawatya, Y. E.; Zeghloul, T.; Dascalescu, L. Influence of Surface Roughness on the Tribo-Electric Process for a Sliding Contact between Polymeric Plate Materials. IOP Conf. Ser.: Mater. Sci. Eng. 2017, 174, 12003.

(50) Cezan, S. D.; Nalbant, A. A.; Buyuktemiz, M.; Dede, Y.; Baytekin, H. T.; Baytekin, B. Control of Triboelectric Charges on Common Polymers by Photoexcitation of Organic Dyes. Nat. Commun. 2019, 10, 276.

(51) Baytekin, H. T.; Baytekin, B.; Soh, S.; Grzybowski, B. A. Is Water Necessary for Contact Electrification? Angew. Chem., Int. Ed. 2011, 50, 6766-6770.

(52) Baytekin, H. T.; Baytekin, B.; Grzybowski, B. A. Mechanoradicals Created in "Polymeric Sponges" Drive Reactions in Aqueous Media. Angew. Chem., Int. Ed. 2012, 51, 3596-3600.

(53) Patil, S. V.; Argyropoulos, D. S. Stable Organic Radicals in Lignin: A Review. ChemSusChem 2017, 10, 3284-3303.

(54) Mazur, T.; Grzybowski, B. A. Theoretical Basis for the Stabilization of Charges by Radicals on Electrified Polymers. Chem. Sci. 2017, 8, 2025-2032.

(55) Gregorova, A.; Košíková, B.; Staško, A. Radical Scavenging Capacity of Lignin and Its Effect on Processing Stabilization of Virgin and Recycled Polypropylene. J. Appl. Polym. Sci. 2007, 106, 16261631. 